Original Article

\title{
A PROSPECTIVE OBSERVATIONAL STUDY ON RISK FACTORS AND MANAGEMENT OF STROKE AT A TERTIARY CARE TEACHING HOSPITAL
}

\author{
MALIHA FATHIMA ${ }^{1}$, SANJEEDA NAJEEB ${ }^{1}$, SUMAIYA FATIMA ${ }^{1}$, SYEDA MASEERA KHALID ${ }^{1}$, SYEDA RANA NIKHAT ${ }^{2}$, \\ RAM CHANDAR RAO ${ }^{3}$
}

1Pharm. D. MESCO College of Pharmacy, Hyderabad, Telangana, India, ²Department of Pharmacology, MESCO College of Pharmacy, Hyderabad, Telangana, India, ${ }^{3}$ Department of General Medicine, Osmania Medical College and Osmania General Hospital, Hyderabad, Telangana, India Email: syedanikhat@yahoo.com

Received: 27 Jan 2018 Revised and Accepted: 27 Apr 2018

ABSTRACT

Objectives: To determine the prevalence of stroke and its management with various risk factors, i.e. non-modifiable and modifiable at a tertiary care teaching hospital.

Methods: It is a prospective and observational study conducted over a period of six months including established cases of stroke. The study was carried out in 100 patients to evaluate risk factors and management of stroke. The data obtained with the designed data collection form were analyzed using Odds ratio and Chi-Square test.

Results: Ischemic stroke accounted for 70\% of cases followed by Intracerebral Hemorrhage (20\%) Subarachnoid hemorrhage (5\%) and Transient Ischemic Attack (5\%). Out of 100 patients, there were 74 male and 26 female patients, indicating males at higher risk. Majority of patients were between the age group 41-60 y. based on the Odds ratio hypertensive and alcoholics were more prone to stroke occurrence and recurrence. ChiSquare test performed for age and gender was not significant at significance level $\mathrm{P}<0.05$.

Conclusion: In this study, ischemic stroke was most prevalent. Hypertension was the major risk factor indicating strong evidence of stroke occurrence and recurrence. The factors having the major implication in the development of stroke were Hypertension, Alcoholism, Smoking, Diabetes Mellitus, Epilepsy, Coronary Artery Disease, Tobacco, and others. Proper management includes non-pharmacological (physiotherapy) along with pharmacological treatment (Osmotic diuretics were most commonly prescribed followed by hypolipidemics, cognition enhancers, anticoagulants, dual antihypertensive therapy).

Keywords: Ischemic stroke, Transient ischemic attack, Intracerebral hemorrhage, Subarachnoid hemorrhage, Hypertension, Alcoholism

(C) 2018 The Authors. Published by Innovare Academic Sciences Pvt Ltd. This is an open access article under the CC BY license (http://creativecommons.org/licenses/by/4.0/) DOI: http://dx.doi.org/10.22159/ijpps.2018v10i6.24983

\section{INTRODUCTION}

Stroke is defined by the World Health Organization as a clinical syndrome consisting of rapidly developing clinical signs of focal (or global in case of coma) disturbance of cerebral function lasting more than $24 \mathrm{~h}$ or leading to death with no apparent cause other than vascular origin [1]. Stroke is becoming an important cause of premature death and disability in low-income and middle-income countries like India. The estimated adjusted prevalence rate of stroke range 84-262/10,000 in rural and 334-424/10,000in urban areas. The incidence rate is 119$145 / 10,000$ based on recent population-based studies there is also a wide variation in case of fatality rates with the highest being $42 \%$ in Kolkata [2]. In China, 1.3 million people have a stroke each year and 75\% live with varying degrees of disability as a result of stroke [3].

Age, gender, race, ethnicity, and heredity have been identified as markers of risk for stroke. Some of the common modifiable risk factors are High blood pressure, Tobacco use, Diabetes, High cholesterol, Atrial fibrillation or another heart disease, Transient ischemic attacks (TIAs), Cigarette Smoking, Alcohol, Migraine. Various lifestyle factors have been associated with increased risk of stroke. These include obesity, physical inactivity, diet, and acute trigger such as emotional stress.

Ischemic stroke (caused by blood clots), Hemorrhagic stroke (caused by ruptured blood vessels that cause brain bleeding) and Transient ischemic attack (TIA) (a "mini-stroke" is a transient episode of neurologic dysfunction caused by ischemia) are three main types of stroke [4].

An ischemic stroke is a sudden neurologic deficit that results from focal cerebral ischemia associated with permanent brain infarction (e. g., positive results on diffusion-weighted MRI).
A transient ischemic attack is focal brain ischemia that causes sudden, transient neurologic deficits and is not accompanied by permanent brain infarction. Intracerebral hemorrhage is focal bleeding from a blood vessel in the brain parenchyma. Subarachnoid hemorrhage is sudden bleeding into the subarachnoid space. The most common cause of spontaneous bleeding is a ruptured aneurysm [5].

The study represents an important resource to progress our understanding of the causes of stroke, both in estimating the contribution of known modifiable risk factors for stroke and in identifying and clarifying the role of new ones, such as genetics. The study was carried out to create awareness among the healthcare professionals and patients regarding its risk factors and management. The health professionals need to promote stroke awareness and provide education to caregivers at discharge and to both survivors and their caregivers after discharge. The objective of present study was to determine the prevalence of stroke and its management with various risk factors, i.e. non-modifiable and modifiable, at a tertiary care teaching hospital.

\section{MATERIALS AND METHODS}

\section{Study design and study duration}

A prospective observational study was conducted after taking Institutional review board approval (MCP/PD/PR/21) in General Medicine, Department of Osmania General Hospital, Hyderabad, India. The study was conducted for a period of six months (December 2016May 2017) on 100 patients diagnosed with a stroke.

\section{Inclusion criteria}

Patients of either sex above $18 \mathrm{y}$ of age diagnosed with stroke, RTA patients with hemorrhage. 


\section{Exclusion criteria}

Pregnant women, HIV or AIDS patient, patients who refused to be the part of the study, patients unable to respond to verbal questions with no caretakers.

\section{Data analysis}

All the relevant and necessary data was collected from: patient's case notes, patients' prescription prescribed by a physician, laboratory report, interviewing the patient or patient caretaker and healthcare professionals. All the patients admitted at Osmania General Hospital were screened for stroke. Each subject's detailed history regarding age, sex, weight, socioeconomic status, rural or urban, history of hypertension, history of stroke and its treatment was given, social habits and any comorbid conditions were collected.

The selected patients were observed for signs and symptoms, the presence of hemorrhage if any, in the cerebrovascular region by CT Scan, motor functioning and improvement in the symptoms following the treatment.

\section{Statistical analysis}

Descriptive statistics were used for the representation of data. Odds Ratio was used to measure the strength of association between the risk factors and the occurrence of stroke. Chi-square test was used to determine the relationship between categorical variables.

\section{RESULTS}

Over a period of six months, a total of 100 patients were included in the study out of which 76 were males and 24 were females.

\section{Incidence of stroke}

The incidence of Ischemic stroke was $70 \%$, accounting for the majority of the cases followed by Intracerebral hemorrhage (20\%) Transient ischemic attack (5\%), Sub-Arachnoid hemorrhage (5\%). The incidence of ischemic stroke was more when compared to other types. The Ischemic stroke was found to be most prevalent in all the age groups. People in the age group 41-60 y are more prone to develop stroke (table 1). Males are more prone to develop all types of stroke when compared to females (table 2). Hypertension is the most common risk factor accounting for 75 cases followed by Alcoholism, Smoking, Diabetes Mellitus and other factors (table 3).

\section{Management \\ Non-pharmacological management}

Patients were asked to undergo regular physiotherapy sessions to remediate impairments and promote mobility, function, and quality of life.

Other non-pharmacological treatment includes frequent position changes and to engage in a passive or active range of motion exercises to strengthen stroke-impaired limbs.

Table 1: Categorization of patients based on age group

\begin{tabular}{lllll}
\hline Age group & Ischemic stroke & Transient ischemic attack & Subarachnoid hemorrhage & Intracerebral hemorrhage \\
\hline $18-40 \mathrm{y}$ & 7 & 1 & 1 & 5 \\
$41-60 \mathrm{y}$ & 30 & 2 & 3 & 11 \\
$>60 \mathrm{y}$ & 31 & 2 & 1 & 4 \\
\hline
\end{tabular}

Table 2: Categorization of patients based on gender

\begin{tabular}{lllll}
\hline Gender & Ischemic stroke & Transient ischemic attack & Subarachnoid hemorrhage & Intracerebral hemorrhage \\
\hline Total & 70 & 5 & 5 & 20 \\
Males & $55(78.5 \%)$ & $4(80 \%)$ & $2(40 \%)$ & $15(75 \%)$ \\
Females & $15(21.42 \%)$ & $1(20 \%)$ & $3(60 \%)$ & $5(25 \%)$ \\
\hline
\end{tabular}

Table 3: Categorization based on risk factors

\begin{tabular}{lll}
\hline S. No. & Risk factors & No. of cases \\
\hline 1 & Hypertension & 75 \\
2 & Alcoholism & 54 \\
3 & Smoking & 43 \\
4 & Diabetes mellitus & 30 \\
5 & Epilepsy & 16 \\
6 & Tobacco chewer & 12 \\
7 & Coronary artery disease & 9 \\
8 & Respiratory tract infections & 4 \\
9 & Electrolyte imbalance & 3 \\
10 & Cardiomyopathy & 2 \\
11 & Pulmonary disease & 2 \\
12 & Meningitis & 2 \\
13 & Atrial fibrillation & 2 \\
14 & A migraine & 1 \\
15 & Psychiatric illness & 1 \\
16 & Cirrhosis of liver & 1 \\
18 & Cancer & 1 \\
\hline
\end{tabular}

\section{Pharmacological management}

The pattern of prescribed drug therapy is given in table 4. In TIA, Osmotic diuretics were most commonly prescribed (100\%), followed by hypolipidemic $(69 \%)$, antiplatelet $(60 \%)$, dual antihypertensive therapy (55\%), cognition enhancers $(20 \%)$ (table 5). In Subarachnoid hemorrhage, Osmotic diuretics were most commonly prescribed (100\%), followed by hypolipidemics $(100 \%)$ calcium channel blocker $(80 \%)$, antianxiety $(40 \%)$, cognition enhancers (40\%) (table 6). In case of intracerebral hemorrhage as well, Osmotic diuretics were most commonly prescribed (90\%), followed by hypolipidemics (65\%), cognition enhancers $(40 \%)$, antianxiety (35), antiplatelet (20\%), calcium channel blockers $(10 \%)$ (table 7$)$. 
Table 4: Drug utilization pattern in ischemic stroke

\begin{tabular}{lll}
\hline S. No. & Class of drug prescribed & \% of drug utilized \\
\hline 1 & Hypolipidemic & $92.8 \%$ \\
2 & Osmotic diuretic & $75.7 \%$ \\
3 & Dual antihypertensive therapy & $75 \%$ \\
4 & Antiplatelets & $71.4 \%$ \\
5 & Cognition enhancers & $31.4 \%$ \\
6 & Anti-anxiety & $17.14 \%$ \\
\hline
\end{tabular}

Table 5: Drug utilization pattern in transient ischemic attack

\begin{tabular}{lll}
\hline S. No. & Class of drug & \% of drug utilized \\
\hline 1 & Osmotic diuretic & $100 \%$ \\
2 & Hypolipidemic & $69 \%$ \\
3 & Antiplatelet & $60 \%$ \\
4 & Dual antihypertensive therapy & $55 \%$ \\
5 & Cognition enhancers & $20 \%$ \\
\hline
\end{tabular}

Table 6: Drug utilization pattern in subarachnoid hemorrhage

\begin{tabular}{lll}
\hline S. No. & Class of drug & \% drug utilized \\
\hline 1. & Osmotic diuretic & $100 \%$ \\
2. & Hypolipidemics & $100 \%$ \\
3. & Calcium channel blocker & $80 \%$ \\
4. & Antianxiety & $40 \%$ \\
5. & Cognition enhancers & $40 \%$ \\
\hline
\end{tabular}

Table 7: Drug utilization in intracerebral hemorrhage

\begin{tabular}{lll}
\hline S. No. & Class of drug & \% of drug utilized \\
\hline 1 & Osmotic diuretic & $90 \%$ \\
2 & Hypolipidemics & $65 \%$ \\
3 & Cognition enhancers & $40 \%$ \\
4 & Anti-anxiety & $35 \%$ \\
5 & Antiplatelets & $20 \%$ \\
6 & Calcium channel blocker & $10 \%$ \\
\hline
\end{tabular}

\section{Statistical analysis}

Apart from descriptive statistics, odds ratio, confidence interval and the significance level were used to measure the strength of association between risk factor and the occurrence of stroke. Based upon odds ratio, hypertension and alcoholism have a significant strength of association between risk factor and the occurrence of stroke (table 8). The chi-square statistic based on age is 5.5739 of $p$-value 0.472572 and the result is not significant at ${ }^{*} p<0.05$ (table 9 ). The chi-square statistic based on gender is 3.8612 of $p$-value 0.276846 and the result is not significant at * $p<0.05$ (table 10).

Table 8: Odds ratio for various risk factors

\begin{tabular}{|c|c|c|c|c|}
\hline S. No. & Risk factors & Odds ratio & Confidence interval & Significance level \\
\hline 1 & Hypertension & 3 & $1.7645-5.1007$ & $\mathrm{P}=0.0001$ \\
\hline 2 & Alcoholism & 1.1739 & $0.7256-1.899$ & $\mathrm{P}=0.5136$ \\
\hline 3 & smoker & 0.7544 & $0.4653-1.2232$ & $\mathrm{P}=0.2530$ \\
\hline 4 & Diabetes Mellitus & 0.4286 & $0.2574-0.7135$ & $\mathrm{P}=0.0011$ \\
\hline 5 & Epilepsy & 0.1904 & $0.1043-0.3478$ & $\mathrm{P}<0.0001$ \\
\hline 6 & Coronary artery disease & 0.0989 & $0.0472-0.2071$ & $\mathrm{P}<0.001$ \\
\hline 7 & Tobacco chewer & 0.1364 & $0.702-0.2648$ & $\mathrm{P}<0.0001$ \\
\hline 8 & Respiratory infections & 0.0417 & 0.1448-0.1176 & $\mathrm{P}<0.001$ \\
\hline 9 & Electrolyte imbalance & 0.309 & 0.0095-0.1008 & $\mathrm{P}<0.0001$ \\
\hline 10 & Cardiomyopathy & 0.0204 & $0.0049-0.0580$ & $\mathrm{P}<0.0001$ \\
\hline 11 & Pulmonary diseases & 0.0204 & $0.0049-0.0580$ & $\mathrm{P}<0.0001$ \\
\hline 12 & Meningitis & 0.0204 & $0.0049-0.0580$ & $\mathrm{P}<0.0001$ \\
\hline 13 & Migraine & 0.0101 & 0.0014-0.0738 & $\mathrm{P}<0.0001$ \\
\hline 14 & Psychiatric Illness & 0.0101 & $0.0014-0.0738$ & $\mathrm{P}<0.0001$ \\
\hline 15 & Cirrhosis Of Liver & 0.0101 & $0.0014-0.0738$ & $\mathrm{P}<0.0001$ \\
\hline 16 & Cancer & 0.0101 & $0.0014-0.0738$ & $\mathrm{P}<0.0001$ \\
\hline 17 & Acute Kidney Injury & 0.0204 & $0.0049-0.0580$ & $\mathrm{P}<0.0001$ \\
\hline 18 & Atrial Fibrillation & 0.0204 & $0.0049-0.0580$ & $\mathrm{P}<0.0001$ \\
\hline
\end{tabular}


Table 9: Chi-square test based on age group

\begin{tabular}{|c|c|c|c|c|c|}
\hline Age group & Ischemic stroke & Transient ischemic attack & Subarachnoid hemorrhage & Intracerebral hemorrhage & Row total \\
\hline $18-40$ & $8(10.5)[0.60]$ & $1(0.75)[0.08]$ & $1(0.75)[0.08]$ & $5(3.00)[1.33]$ & 15 \\
\hline $41-60$ & $31(32.90)[0.12]$ & $2(2.35)[0.05]$ & $3(2.35)[0.18]$ & $11(9.40)[0.27]$ & 47 \\
\hline$>60$ & $31(26.60)[0.73]$ & $2(1.90)[0.01]$ & $1(1.90)[0.43]$ & $4(7.60)[1.71]$ & 38 \\
\hline column totals & 70 & 5 & 5 & 20 & 100 \\
\hline
\end{tabular}

Table 10: Chi-square test based on gender

\begin{tabular}{|c|c|c|c|c|c|}
\hline Gender & Ischemic stroke & Transient ischemic attack & Subarachnoid hemorrhage & Intracerebral hemorrhage & Row total \\
\hline Male & $55(53.2)[0.06]$ & $4(3.80)[0.01]$ & $2(3.80)[0.85]$ & $15(15.20)[0.00]$ & 76 \\
\hline Female & $15(16.80)[0.19]$ & $1(1.20)[0.03]$ & $3(1.20)[2.70]$ & $5(4.80)[0.01]$ & 24 \\
\hline column totals & 70 & 5 & 5 & 20 & 100 \\
\hline
\end{tabular}

\section{DISCUSSION}

In present study evidence of stroke was observed in 100 patients, the incidence of ischemic stroke was found out to be the highest which was consistent with Chachu kuriakose et al. (2016) [6]. Hypertension, Diabetes mellitus, Coronary artery disease, cigarette smoking, tobacco chewer, epilepsy, etc., were all found to have a high risk for stroke occurrence.

The stroke cases that were collected in our study, accounted for 76 males and 24 females, which gives a clear idea that the occurrence of stroke is more common in males compared to females, which was in accordance with Laxman Wagle et al. (2017) [7]. This may be due to heavy alcohol consumption and cigarette smoking in males. It was also found that ischemic stroke is the most prevalent of all stroke types in both the genders.

Age is also considered as one of the risk factors in our study. The results that were obtained showed that patients in the age group of 41-60 y accounted a larger number followed by geriatrics, the age above $60 \mathrm{y}$. Again ischemic stroke was the most prevalent of all types of stroke.

The various risk factors were observed and their association with stroke was analyzed. There has not been a single study before that included so many risk factors which are the major strength of our study.

The most prevalent risk factor in the present study was found to be hypertension, out of 100 patients, 75 patients were hypertensive, which was in accordance with Sahbanathul missriya et al., (2017) [8]. Thus, maintaining BP within normal range was found to be helpful to prevent stroke occurrence and recurrence. This was found consistent with Parveen et al., (2003) [9], which reported that overall lowering BP was associated with significant reduction in all types of stroke. It was reported from previous studies that recurrent stroke was reduced when BP was controlled at $<120 / 80 \mathrm{~mm}$ of $\mathrm{Hg}$, conducted by Gary et al. (2003) [10]. Our results also imply for BP that "lower is better" as a goal in reducing stroke recurrence risk, which was found to be consistent with the study of Gary et al. (2003) [10].

The patients who were given dual antihypertensive therapy were at lower risk of developing stroke and patients newly diagnosed with stroke were managed better when compared to monotherapy. Our study found to be consistent with studies conducted by Parveen et al. (2003) [9] wherein they reported that reduction in stroke events was simply related to the magnitude of BP reduction an assertion supported by PROGRESS trial, in which Dual therapy was superior to monotherapy in lowering both BP and Stroke risk.

Alcoholism is another important risk factor having a high incidence in our study, out of 100 patients, 54 were alcoholic among which majority were heavy drinkers which indicated an increased risk of all stroke types. Our study was found to be in accordance with Larsson et al. (2016) [11], which reported that heavy alcohol consumption was associated with an increased risk of all stroke types but with a stronger association with hemorrhagic type.

Smoking is the third most prevalent risk factor accounting for 43 patients out of 100 patients. It was found that chronic cigarette smokers were at a higher risk of developing stroke compared to non-smokers, this was in accordance with Robert et al. (1986) [12], where they found out that cigarette smokers had 2-3 times the risk of thromboembolic or hemorrhagic stroke, after controlling for age, DBP, CHD and other risk factors. In our study, we also found out that former smokers are also at a risk of developing stroke.

About 30 patients out of 100 were found to have DM, indicating that it is another risk factor associated with both stroke occurrence and recurrence. Kothari et al. (2002) [13], people with type II DM are at elevated risk of stroke compared with those without DM.

In the present study, patients with an increase in cholesterol levels were significantly associated with stroke. It was also found that HDL was protective against stroke which was inconsistent with Thomas et al. (2003) [14], which states, HDL may be protective against stroke, whereas, elevated levels of LDL, TC may pose as a risk factor for the development of stroke.

Restroke was observed in 26 patients out of 100 . Mostly, in patients with elevated BP and also in patients with more than one comorbidity. Alcoholism and cigarette smoking also play an important role in developing restroke.

It was found that proper management at the proper time can be lifesaving. In delayed hospital admission, thrombolytic therapy is not effectively reported by Tapas Kumar et al. (2016)[2].

Osmotic diuretics were most commonly prescribed, followed by hypolipidemics, cognition enhancers, anticoagulants, etc. Osmotic diuretics were administered in almost all patients and were found to be beneficial in reducing ICP, thus helping to prevent further worsening of the condition.

Based on the comorbid conditions the required treatment was provided and was found to be beneficial. Physiotherapy sessions were conducted in patients whose overall condition has been stabilized and it was found to be beneficial in improving the mobility of the patient.

\section{CONCLUSION}

The present study has focused on risk factors and management of stroke, ischemic stroke was found to be more prevalent. The risk factors play an important role in the development and progression of the disease. Among non-modifiable risk factors, males of age group 41-60 y were more prone to stroke occurrence and stroke recurrence was more prevalent in the age group above $60 \mathrm{y}$. Among the modifiable risk factors, Hypertension accounted for $75 \%$ of the cases clearly indicating strong evidence of elevated BP levels with stroke occurrence and recurrence.

The following factors had an implication in the development of stroke in following order:

Hypertension $>$ Alcoholism $>$ Smoker $>$ DiabetesMellitus $>$ Epilepsy $>$ CAD $>$ Tobacco $>$ Respiratory Infections $>$ Electrolyte Imbalance $>$ Pulmonary Diseases $=$ Meningitis $=$ Cardiomyopathy $=$ Atrial Fibrillation $=$ Acute Kidney Injury $>$ Migraine $=$ Psychiatric Illness $=$ Cirrhosis of Liver $=$ Cancer

The goal of therapy should be to treat co-morbid conditions which will help to lessen the risk for the development of restroke. Proper management includes non-pharmacological interventions along with pharmacological treatment. 


\section{AUTHORS CONTRIBUTIONS}

It was a collective work of all the authors. Maliha Fathima (Pharm D), Sanjeeda Najeeb (Pharm. D), Sumaiya Fatima (Pharm. D), Syeda Maseera Khalid (Pharm. D) were responsible for literature review, data collection, data entry, data analysis, manuscript writing. Ms. Syeda Rana Nikhat contributed for data analysis, manuscript writing along with other authors and responsible for communication.

\section{LIMITATIONS}

The limitation of our study includes small sample size and short duration of the study.

\section{CONFLICT OF INTERESTS}

The authors declare no competing financial interest

\section{REFERENCES}

1. https;//www.ncbi.nlm.nih.gov/books/NBK53302/. accessed on 20 Dec 2017

2. Tapas Kumar Banerjee, Shyamal Das. Fifty years of stroke researches in India. Ann Indian Accad Neurol 2016;19 suppl 1:11-8.

3. www.stroke.ahajournals.org. [Last accessed on 20 Dec 2017]

4. www.cdc.gov/stroke/typesofstroke.html. [Last accessed on 20 Dec 2017].

5. http://www.merckmanuals.com/professional/neurologicdisor ders/stroke/transient-ischemic-attack-tia. [Last accessed on 20 Dec 2017].
6. Chachu Kuriakose, Naseem Shifafiya M, Nelta S Tharakan, Sattanathan K, Sambath Kumar R. A prospective study of clinical profile of stroke in a tertiary care hospital. Asian J Pharm Clin Res 2016;9:178-81.

7. Laxman Wagle, Abel Abraham Thomas, Sunil Shrestha. Drug utilisation study of stroke and other patients admitted to general ward of neurology unit at quaternary care private hospital. Int J Curr Pharm Res 2017;9:23-8.

8. Sahbanathul Missriya MA, Johncey John. Assess the prevalence of hypertension and knowledge regarding the prevention of stroke. Asian J Pharm Clin Res 2017;10:177-80.

9. Parveen Rashid, Jo Leonardi Bee, Philip Bath. Blood pressure reduction and secondary prevention of stroke and other vascular events a systematic review. Stroke 2003;34:2741-48.

10. Gary Friday, Milton Alter, Sue Min Lai, J0 Connell, E Sobel. Hypertension and risk of stroke recurrence. Stroke 2002;33:2652-7.

11. Larsson SC, Wallin A, Wolk A, Markus HS. Differing association of alcohol consumption with different stroke types: a systematic review and meta analysis. BMC Med 2016;14:178.

12. Abbott RD, Yin Y, Reed DM, Yano K. Risk of stroke in male cigarette smokers. N Engl J Med 1986;18:717-20.

13. Viti Kothari, Richard J Stevens, Amanda I Adler, Irene M Stratton, Susan E Manley, H Andrew Neil, et al. Risk of stroke in type 2 diabetes estimated by the UK prospective diabetes study risk engine. Stroke 2002;33:1776-81.

14. Thomas S Bowman, Howard D Sesso, Jing Ma, Tobias Kurth Carlos S Kase, Meir J Stumpfer, et al. Cholesterol and the risk of ischemic stroke. Stroke 2003;34:2930-4 